\title{
Rechtsprechung
}

\section{Restitutionsansprüche der Erben eines jüdischen Kunsthändlers}

\author{
Oberlandesgericht Köln, Urteil vom 8. Juli 2016 - 1 U 36/13
}

Zu den Restitutionsansprüchen der Erben eines jüdischen Kunsthändlers, der aufgrund des Verfolgungsdrucks des NS-Regimes im Jahr 1937 zum Verkauf seines Galeriebestandes gezwungen war (amtlicher Leitsatz).

\section{Gründe}

I.

- 1. Mit der Klage begehrt die Klägerin Schadensersatz für den Verlust des Gemäldes „Der heilige Hieronymus mit dem Löwen und zwei Engeln“ des Malers D. Das Bild war im Jahr 1937 durch den Rechtsvorgänger der Beklagten versteigert worden. Das Gemälde befand sich zum damaligen Zeitpunkt im Eigentum des jüdischen Kunsthändlers Dr. T (fortan: Erblasser). Das NSRegime hatte im Zusammenhang mit der Judenverfolgung dem Erblasser die ausgeübte Tätigkeit als Kunsthändler untersagt und ihn so zum Verkauf seiner Gemälde gezwungen. Nach dem Krieg verlangte der Erblasser vom Land Nordrhein-Westfalen auf Grundlage des Bundesentschädigungsgesetzes (BEG, Bundesgesetz zur Entschädigung für Opfer der nationalsozialistischen Verfolgung) hinsichtlich der Kunstwerke, die im Jahr 1937 durch den Rechtsvorgänger der Beklagten versteigert worden waren, Entschädigung für den Schaden, der ihm durch die verfolgungsbedingte Verschleuderung seiner Werke entstanden war. In der Klageschrift vom 6. Dezember 1962 (Blatt $107 \mathrm{ff}$ der Akte), mit welcher der Erblasser als Ersatz für den Verschleuderungsschaden einen Betrag von 10.066,41 DM (Antrag zu 1) und als Ersatz des ihm verfolgungsbedingt entstandenen Steuerschadens einen Betrag von 12.311,51 DM (Antrag zu 2) verlangte, ließ er unter anderem vortragen:

„... Ein Vermögensschaden wird auch nicht dadurch ausgeräumt, dass das Kunsthaus $M$ versichert hat, dass die Bilder zu der damaligen Marktlage entsprechenden Preisen versteigert worden sind und dass der Kläger bei der Vorbereitung der Versteigerung selbst mitgewirkt hat, indem er dem Kunsthaus M Mindestpreise vorgeschrieben hat.

Dabei soll vorweg nochmals hervorgehoben werden, dass gegen die Inhaber des Kunsthauses $M$ keine persönlichen Vorwürfe erhoben wurden. [...] Ob ein Verschleuderungsschaden eingetreten ist oder nicht, hängt nicht von den Umständen ab, unter denen es zur Versteigerung gekommen ist. Entscheidend ist nach unserer Auffassung einzig und allein die Frage, ob der Verkauf der Bilder in den Räumen der Galerie unter verfolgungsunabhängigen Umständen denselben Erlös erbracht hätte, der bei der Versteigerung erzielt worden ist, oder ob bei der Versteigerung ein wesentlich niedrigerer Erlös erzielt worden ist...."
Durch Teilurteil vom 24. Februar 1964 verurteilte die 7. Entschädigungskammer des LG Düsseldorf - 26 O(Entsch) 454/62 - das Land Nordrhein-Westfalen an den Erblasser einen Betrag in Höhe von 8.581,50 DM zu zahlen. Zu den Werken, hinsichtlich derer der Erblasser einen Verschleuderungsschaden geltend machte, gehörte auch das nämliche Bild „Der heilige Hieronymus mit dem Löwen und zwei Engeln" des Malers D. Dieses hatte der vom Erblasser beauftragte Sachverständige Q (Blatt 287 ff der Akte, dort Blatt 294 der Akte), dem auch die Entschädigungskammer des LG Düsseldorf in ihrer Entscheidung gefolgt war, durch einen Vergleich des vom Erblasser angegebenen Versteigerungserlöses mit einem möglichen Verkaufspreis, wie er in einem normalen Geschäftsbetrieb eines Kunsthauses im Jahre 1937 erzielt worden wäre. Dabei hat der Sachverständige offensichtlich irrtümlich - wie sich aus der Klage des Erblassers ergibt - DM-Werte anstatt Reichsmark-Werten geschrieben, aber im folgenden richtig gerechnet:

\begin{tabular}{|c|c|c|c|c|c|}
\hline Nr. & Gemälde & $\begin{array}{c}\text { Versteige- } \\
\text { rungserlös }\end{array}$ & $\begin{array}{c}\text { Ausgez. } \\
\text { Betrag }\end{array}$ & $\begin{array}{c}\text { Möglicher } \\
\text { Verkaufs- } \\
\text { wert }\end{array}$ & $\begin{array}{c}\text { Differenz- } \\
\text { Betrag }\end{array}$ \\
\hline 185. & $\begin{array}{c}\text { L. D 40/31,5 } \\
\text { Heil. } \\
\text { Hieronymus }\end{array}$ & DM 4800,- & DM 4320,-- DM 6000,- & DM 1680,-- \\
\hline
\end{tabular}

Das LG Düsseldorf ging bei seiner Entscheidung unter anderem von folgendem Tatbestand aus:

„Der am 18.4.1904 in N geborene Kläger ist Jude. Er war Inhaber der Gemäldegalerie T2 in E. Im Sommer 1937 wurde ihm aus Verfolgungsgründen untersagt, seine Kunsthandlung weiter zu betreiben. Er ließ deshalb am 13.11.1937 den größten Teil der Bilder seiner Galerie durch das Kunsthaus M in L versteigern. Nach seiner Erinnerung betrug der Versteigerungserlös nach Abzug der Unkosten 55.000,- RM (Blatt 46 der Entschädigungsakte)...."

Gegen die vorgenannte Entscheidung legte der Erblasser kein Rechtsmittel ein. Hinsichtlich des mit dem Klageantrag zu 2) geltend gemachten und durch das Teilurteil nicht beschiedenen Steuerschadens schlossen das Land Nordrhein-Westfalen und der Erblasser am 11. Oktober 1965 einen Vergleich, durch welchen sich das genannte Land zur Zahlung eines Betrages in Höhe von 6.300 DM und zur Übernahme von 3/5 der außergerichtlichen Kosten des Erblassers verpflichtete. 
Im Jahr 2000 schließlich bot die Beklagte zu 1) das nämliche Gemälde erneut in einer Auktion an. Im Katalog der Versteigerung vom 20. Mai 2000 heißt es zur Provenienz:

„Privatsammlung, C (1933); Galerie T2, E; 392. M Auktion L, 13.11.1937, Lot 185 (die Bestände der Galerie T2, E); rheinische Privatsammlung; $A . "$

Am 18.05.2000 ließ die Beklagte zu 1) auf Nachfrage der Klägerin per Fax (Anlage K 4, Blatt 15 der Akte) unter anderem Folgendes erklären:

"The provenance and history of the painting is clean. We sold it 1937 (Die Bestände der Galerie T2, E) to a collector in the Rhine area (near L)."

Als die Klägerin um eine Bestätigung bat, dass das Art Loss Register konsultiert worden war und es kein Problem hinsichtlich Diebstahls, Konfiszierung oder Zwangsverkauf gebe, ließ der Beklagte zu 2) mitteilen, das Art Loss Register kontrolliere den Katalog. Das Art Loss Register ist die weltweit größte Datenbank verlorener und gestohlener Kunstwerke. Vor diesem Hintergrund ersteigerte die Klägerin das Gemälde zu einem Gebot von 100.000 DM zuzüglich 16.000 DM Aufgeld und ließ es am 31. Mai 2000 nach New York transportieren. Als die Zeitung "New York Times“ im April 2009 einen Beitrag über die Restitution eines anderen Gemäldes veröffentlichte, das sich ebenfalls im Eigentum der Galerie T2 befunden hatte und im Jahr 1937 in derselben Auktion wie das streitgegenständliche Bild versteigert worden war, stellte die Klägerin Nachforschungen an und kontaktierte das Art Loss Register. Hierbei erfuhr sie, dass das von ihr ersteigerte Gemälde seit dem Jahr 2004 als vom NS-Regime gestohlen gemeldet ist. Der nachfolgend kontaktierte Leiter des „T Restitution Project", welches sich um die Wiederbeschaffung der zwangsverkauften Kunstwerke des Erblassers bemüht, bestätigte, dass das Gemälde 1937 durch den Rechtsvorgänger der Beklagten versteigert worden war und informierte das Heimatschutzministerium in den USA von dem Sachverhalt. Dieses wandte sich umgehend an die Klägerin, um einen Beschlagnahmeanspruch der Vereinigten Staaten geltend zu machen und die Herausgabe des Bildes zu verlangen. Am 27./28. Mai 2009 unterzeichnete der Geschäftsführer der Klägerin eine "Stipulation“ (Vereinbarung), in der er der Beschlagnahme durch die amerikanischen Behörden zustimmte und die Herausgabe des Gemäldes an die Erben vereinbarte (Anlage K12, Blatt 36 ff der Akte). Das Gemälde wurde den USBehörden übergeben, die es an die Erben von Dr. T zurückgaben. In der Stipulation heißt es unter anderem (vgl. auch die von der Klägerin als Anlage K 12 vorgelegte nicht amtliche Übersetzung, Blatt $41 \mathrm{f}$ der Akte):

„... Nach dem Zwangsverkauf floh Dr. T aus Deutschland und ließ sich schließlich in Kanada nieder. Die Naziregierung verhinderte, dass er die Auktionserlöse erhielt. Während des zweiten Weltkrieges wurden viele Unterlagen des LAH (Anm: Auktionshaus M) zerstört, was nach dem Krieg die Suche und Identifizierung von
Käufern der Sammlung von Dr. T behinderte. Nichtsdestotrotz unternahm Dr. T Versuche, die ihm gestohlenen Kunstwerke seiner Sammlung zu finden...."

Die Klägerin hat behauptet, der Beklagte zu 2) habe in einem Telefonat am 17. Mai 2000 garantiert, dass es mit Blick auf die Auktion von 1937 keine Probleme mit dem Bild gebe. Überdies habe er angegeben, bei der Galerie T2 habe es sich nicht um eine jüdische Galerie gehandelt. Die Klägerin ist der Ansicht gewesen, das Gemälde sei bei der Auktion im Jahr 2000 mit einem zum Schadensersatz berechtigenden Rechtsmangel behaftet gewesen. Sie hat deshalb beantragt,

die Beklagten zu verurteilen, als Gesamtschuldner an sie USD 350.000,00 nebst Zinsen in Höhe von 8 Prozentpunkten über dem Basiszinssatz seit dem 2. Juni 2012 zu zahlen.

Die Beklagten haben beantragt,

die Klage abzuweisen.

Sie haben behauptet, die Klägerin sei nach amerikanischem Recht nicht verpflichtet gewesen, das Gemälde zurückzugeben. Im Übrigen sind sie der Ansicht gewesen, ein ausländisches Recht könne keinen Rechtsmangel begründen. Sie haben die Einrede der Verjährung erhoben.

2. Das Landgericht hat die Klage abgewiesen (Urteil in KUR 5/2013 S. 154ff)

3. Hiergegen richtet sich die Berufung der Klägerin. Es bestehe ein materiell-rechtlicher Herausgabeanspruch der Erben und ein behördlicher Beschlagnahmeanspruch nach New Yorker Recht. Dies stelle zugleich einen Rechtsmangel nach deutschem Recht dar. Dies gelte für ausländische Rechte Dritter unabhängig davon, ob es eine Entsprechung im deutschen Recht gebe, wobei es ungeachtet dessen auch eine derartige Entsprechung in der deutschen Rechtsordnung bestehe. Auch sei der Anspruch nicht verjährt. Die Verjährungsfrist habe nicht bereits im Jahr 2000 sondern erst mit Herausgabe des Gemäldes im Jahr 2009 zu laufen begonnen. Entscheidend sei im vorliegenden Fall die Sichtweise des amerikanischen Rechts. Die Stipulation und die Bissonnette-Urteile würden darauf schließen lassen, dass das zuständige amerikanische Gericht den Grundsatz der lex rei sitae unter Berufung auf den amerikanischen ordre public nicht anerkennen würde. Auch sei nach dem New-Yorker Recht keine Verjährung eingetreten, weil diese erst mit der Ablehnung des Herausgabeverlangens beginne.

\section{Die Klägerin beantragt,}

die Beklagten werden in Abänderung des Urteils des LG Köln vom 24. April 2013 - 23 O 266/12 - verurteilt, als Gesamtschuldner 284.830,73 € nebst Zinsen in Höhe von 8 Prozentpunkten über dem Basiszinssatz seit dem 2. Juni 2012 an die Klägerin zu zahlen. 
Die Beklagten beantragen,

die Berufung zurückzuweisen.

Sie verteidigen unter Wiederholung und Vertiefung ihres erstinstanzlichen Vorbringens die angefochtene Entscheidung. Eine Rechtsmangelhaftung wegen Nichterfüllung scheitere, weil der Beklagte zu 2) der Klägerin gemäß § 929 S. 1, § 932, § 935 Abs. 2 BGB nach dem gemäß Art. 43 Abs. 1 EGBGB anzuwendenden deutschen Recht im maßgeblichen Zeitpunkt der Übergabe an den Transporteur Eigentum verschafft habe und zwar frei von Rechten Dritter. Letzteres ergebe sich bereits aus $\S 936$ Abs. 1 BGB. Der Statutenwechsel, der nach der Übergabe des Gemäldes an den Spediteur durch den Transport nach New York eingetreten sei, führe zu keinem anderen Ergebnis. Die Beklagten hafteten nur bis zur Übergabe an den Spediteur. Wegen Art. 43 Abs. 2 EGBGB komme es nicht darauf an, ob die Erben von Dr. T nach amerikanischem Recht als Eigentümer des Gemäldes anzusehen seien, weil der dingliche Eigentumserwerb der Klägerin noch im Erststaat - Deutschland - abgeschlossen worden sei. Selbst wenn auf eine Klage der Erben gegen die Klägerin abzustellen sei, sei nicht amerikanisches sondern kanadisches Recht der Provinz Québec anzuwenden. Es liege ein Fall der sog. Nachlassspaltung vor, bei dem sich die Erbfolge in bewegliches Eigentum nach dem Recht des letzten Wohnsitzes des Erblassers - hier Montreal - richte. Doch auch wenn eine kollisionsrechtliche Anknüpfung mit dem amerikanischen Recht gesehen werde, sei nicht das Recht des Staates New York, sondern das Recht des Staates Illinois anzuwenden, weil die Klägerin als Kapitalgesellschaft dort registriert sei. Doch auch wenn das Recht des Staates New York anzuwenden sei, folge aus den sog. Bissonnette-Urteilen kein Beleg dafür, dass eine Replevin-Klage der Erben Aussicht auf Erfolg gehabt hätte. Auf den amerikanischen ordre public komme es nicht an, weil der deutsche Richter nicht das amerikanische IPR, sondern ausschließlich das deutsche IPR zu prüfen habe. Auch die Beschlagnahme durch das U.S. Department of Justice stelle keinen Rechtsmangel dar. Selbst wenn man einen Rechtsmangel annehme, fehle es jedenfalls an der gemäß § 326 Abs. 1 BGB a.F. erforderlichen Nachfristsetzung, weil es sich um einen Fall des Verzuges handele. Erst durch die freiwillige Herausgabe der Klägerin an die US-Behörden sei Unmöglichkeit eingetreten, was diese zu vertreten habe. Überdies berufen die Beklagten sich auf Verwirkung und Verjährung.

Der Senat hat Beweis erhoben durch Einholung eines schriftlichen Gutachtens des Sachverständigen Prof. Dr. N2 zur Rechtslage nach US-amerikanischem Recht, insbesondere zu der Frage, ob nach dem Recht des Staates New York am 31. Mai 2000 ein Anspruch der Erben des Dr. T gegen die Klägerin auf Herausgabe des nämlichen Gemäldes bestanden habe und ob dieses von den US-Behörden in New York zum genannten Zeitpunkt habe rechtmäßig beschlagnahmt werden können. Die Akte 26 O (Entsch.) 454/62 LG Düsseldorf war beigezogen und Gegenstand der mündlichen Verhandlung.
II.

Die zulässige Berufung ist unbegründet. Das Landgericht hat im Ergebnis zutreffend entschieden. Die Klägerin kann von den Beklagten unter keinem rechtlichen Gesichtspunkt die Zahlung von Schadensersatz verlangen.

1. Die Klägerin hat gegen die Beklagten keinen Anspruch auf Zahlung von Schadensersatz aus § 434, § 440, § 325 Abs. 1 BGB in der vor dem 1. Januar 2002 geltenden Fassung. Diese ist maßgeblich, weil für den hier vorliegenden Fall, in dem das Schuldverhältnis vor dem 1. Januar 2002 entstanden ist, das Bürgerliche Gesetzbuch gemäß Art. $229 \S 5$ EGBGB in der bis zu diesem Tag geltenden Fassung Anwendung findet. Das Gesetz zur Modernisierung des Schuldrechts vom 26. November 2001 (BGBI. I 3138) findet noch keine Anwendung. Gemäß § 434 $B G B$ in der genannten Fassung war der Verkäufer verpflichtet, dem Käufer die Sache frei von Rechten zu verschaffen, die von Dritten gegen den Käufer geltend gemacht werden können. Unerheblich ist dabei, ob der Dritte sein Recht erst nach Gefahrübergang ausübt; der Verkäufer ist verpflichtet, schon die bloße Gefahr der Inanspruchnahme zu beseitigen (BGH, Urteil vom 18. Februar 2004 - VIII ZR 78/03, NJW 2004, 1802, zitiert juris Rn. $8 \mathrm{mwN}$ ). Unter die Rechte Dritter im Sinne des $\S 434$ BGB a.F. fallen nicht nur dingliche oder obligatorische Privatrechte sondern auch öffentlich-rechtliche Befugnisse, wobei dies auch für ausländische Ansprüche und Befugnisse gilt (vgl. BGH, Urteil vom 7. Oktober 1991 - II ZR 252/90, NJW 1992, 362, zitiert juris Rn. 9; OLG Köln, Beschluss vom 16. März 2010 - 22 U 176/09, zitiert juris Rn. 6; OLG Düsseldorf, Urteil vom 20. Februar 2015 - 22 U 159/14, zitiert juris Rn. 4; Palandt/Weidenkaff, BGB, 75. Aufl., § 435 Rn. 8; Staudinger/Matusche-Beckmann, BGB, § 435 Rn. 12). Allerdings begründet die Beschlagnahme durch eine staatliche Behörde einen Rechtsmangel nur dann, wenn diese - etwa wie eine Beschlagnahme nach §§ 111b und c StPO - den Verfall oder die Einziehung der Sache zur Folge haben kann (vgl. BGH, Urteil vom 18. Februar 2004, aaO Rn. 10; OLG Köln, Beschluss vom 16. März 2010, aaO). Gemessen hieran fehlt es an einem Rechtsmangel des nämlichen Kunstwerkes bei Gefahrübergang. Ansprüche Dritter bestehen weder nach deutschem noch nach US-amerikanischen Recht.

a) Es besteht kein Anhalt dafür, dass die Klägerin nach deutschem Recht kein Eigentum erworben hat. Dass der Vollzug des Kaufvertrags stecken geblieben wäre, weil die Klägerin nach deutschem Recht kein Eigentum an dem Gemälde erworben hat, macht diese schon nicht geltend. Überdies würde dies auch nicht zu einem Rechtsmangel führen. Vielmehr hätte der Verkäufer seine Pflicht zur Übereignung der verkauften Sache nach § 433 Abs. 1 Satz 1 BGB nicht erfüllt; die fehlende Verschaffung des Eigentums stellt keinen Rechtsmangel nach § 435 BGB dar (BGH, Urteil vom 19. Oktober 2007 - V ZR 211/06, BGHZ 174, 61 Rn. 27; MünchKomm-BGB/Westermann, 6. Aufl., § 435 Rn. 7; jeweils $\mathrm{mwN}$ ). Davon abgesehen ist die Klägerin auch Eigentümerin des Gemäldes geworden. Für den Eigentumserwerb gilt das Recht der belegenen Sache (lex rei sitae) gemäß Art. 43 
Abs. 1 EGBGB und damit das deutsche Recht. Danach hat die Klägerin, wie das Landgericht rechtsfehlerfrei ausgeführt hat, wegen § 935 Abs. 2 BGB selbst dann gutgläubig Eigentum an dem Gemälde erlangt, wenn dieses als abhandengekommen zu bewerten sein sollte, weil das Kunstwerk im Wege öffentlicher Versteigerung veräußert wurde. Ein Herausgabeanspruch der Erben gemäß § 985, § 1922 BGB ist daher nicht gegeben.

Anderweitige Herausgabeansprüche der Erben Dr. Ts nach deutschem Recht sind ebenfalls nicht ersichtlich. Ansprüche nach dem Bundesentschädigungsgesetz (BEG) hat der Erblasser im Verfahren 26 O (Entsch.) 454/62 LG Düsseldorf geltend gemacht und dabei vom Land Nordrhein-Westfalen einen Ersatz des Verschleuderungs- und des Steuerschadens erhalten. Soweit Entschädigungsgesetzte im Übrigen (Kulturgüterrückgabegesetz [KultGüRückG], Bundesrückerstattungsgesetz [BRüG], Gesetz zur Regelung offener Vermögensfragen [VermG] etc.) bestehen, wurden etwaige Ansprüche, soweit die genannten Gesetze überhaupt einschlägig sind, von dem Erblasser beziehungsweise seinen Erben nicht und damit auch nicht rechtzeitig angemeldet. Restitutionsabkommen wie die Washingtoner Erklärung von 1998 sind unabhängig von ihrer Anwendbarkeit rechtlich nicht bindend und begründen keine individualrechtlichen Rückgabeansprüche (vgl. VG Magdeburg, Urteil vom 17. Januar 2012 - 7 A 326/10, zitiert juris Rn. 54; VG Berlin, Urteil vom 29. November 2006 - 1 A 162.05, zitiert juris Rn. 41). Gleiches gilt für die „Erklärung der Bundesregierung, der Länder und der kommunalen Spitzenverbände zur Auffindung und zur Rückgabe NS-verfolgungsbedingt entzogenen Kulturgutes, insbesondere aus jüdischem Besitz“ vom Dezember 1999 (VG Berlin, aaO).

b) Ein Rechtsmangel hinsichtlich des nämlichen Kunstwerkes bei Gefahrübergang folgt auch nicht aus einem Herausgabeanspruch der Erben des Erblassers nach US-amerikanischem Recht. Dies gilt unabhängig davon, ob ein New Yorker Gericht sich für eine gegen die Klägerin gerichtete Klage auf Herausgabe des nämlichen Kunstwerkes für zuständig erklärt und es auf Grundlage des am Ort des angerufenen Gerichts geltenden Rechts (lex fori) entschieden hätte.

Zwar können auch ausländische Rechte Dritter einen Rechtsmangel nach deutschem Recht begründen (vgl. BGH, Urteil vom 7. Oktober 1991 - II ZR 252/90, NJW 1992, 362, zitiert juris Rn. 9; OLG Köln, Beschluss vom 16. März 2010 - 22 U 176/09, n.v., zitiert juris Rn. 6; Palandt/Weidenkaff, BGB, 75. Aufl., § 435 Rn. 8). Für den Senat wäre insoweit der Umstand maßgeblich, dass die Klägerin als Gesellschaft mit Sitz im Staat New York für die Beklagte ersichtlich das bei dieser ersteigerte Kunstwerk in die USA ausführen wollte. Es bedarf in diesem Zusammenhang auch keiner Entscheidung, ob das zuständige amerikanische Gericht den Grundsatz der lex rei sitae unter Berufung auf den amerikanischen ordre public anerkennen würde. Für diese Annahme erfordert es überdies keines Rückgriffs auf das deutsche Recht als das lex fori (vgl. BGH, Beschluss vom 26. Oktober 1977 - IV ZB 7/77, BGHZ 69, 387, zitiert juris Rn. 20; vom 23. Dezember
1981 - IVb ZR 643/80, NJW 1982, 1215, zitiert juris Rn. 21), oder einer Anwendung des nächst verwandten Rechts als Ersatzrecht (vgl. BGH, Urteil vom 23. Dezember 1981, aaO; MünchKommZPO/Prütting, 4. Aufl., § 293 Rn. 59; Hk-ZPO/Saenger, 5. Aufl., § 293 Rn. 22; jeweils mwN) mit der Begründung, das ausländische Recht lasse sich nicht zuverlässig ermitteln. Denn auch nach USamerikanischen Recht, hier dem Recht des Staates New York, besteht kein gegen die Klägerin gerichteter Anspruch der Erben auf Herausgabe des nämlichen Kunstwerkes. Dieses Recht hatte der Senat wegen § 293 ZPO von Amts wegen und unter Berücksichtigung seiner konkreten Ausgestaltung in der ausländischen Rechtspraxis, insbesondere der ausländischen Rechtsprechung, zu ermitteln (vgl. BGH, Urteil vom 30. April 1992 - IX ZR 233/90, BGHZ 118, 151, zitiert juris Rn. 25; vom 23. Juni 2003 - II ZR 305/01, NJW 2003, 2685, zitiert juris Rn. 9; Beschluss vom 21. Dezember 2011 - I ZR 144/09, TranspR 2012, 110, zitiert juris Rn. $11 \mathrm{ff}$ ). Die Parteien trifft insoweit keine (prozessuale) Beweisführungslast (BGH, Beschluss vom 21. Dezember 2011, aaO Rn. 11).

aa) Auf Grundlage der Ausführungen des Sachverständigen, die der Senat nachvollzieht und sich zu eigen macht, ist allerdings davon auszugehen, dass ein New Yorker Gericht sich für eine gegen die Klägerin gerichtete Klage auf Herausgabe des nämlichen Kunstwerkes für zuständig erklärt und hierüber entschieden hätte. Ein Gericht in den USA darf einen Rechtsstreit nur entscheiden, wenn es in persönlicher und sachlicher Hinsicht Zuständigkeit besitzt (vgl. hierzu Rn. 235 des Gutachtens $m w N$ ). Die Zuständigkeit in persönlicher Hinsicht (personal jurisdiction) beruht bei der beklagten Partei auf einer ausreichenden Verbindung zu dem Staat des urteilenden Gerichts. Bei einer juristischen Person wird eine ausreichende wesentliche und dauerhafte Beziehung zu einem Staat durch ihre Gründung in diesem Staat hergestellt; ausreichend ist aber auch, dass sie im Forumstaat als geschäftstreibend eingetragen und berechtigt ist, Geschäfte zu betreiben (Rn. 236 und 238 des Gutachtens mwN). Im Recht des Staates New York folgt dies aus Sec. 301 CPLR (Jurisdiction over persons, property or status): „A court may exercise such jurisdiction over persons, property, or status as might have been exercised heretofore." So liegt der Fall auch hier. Es ist zwischen den Parteien unstreitig, dass die Klägerin ihre Geschäfte in New York betreibt. Da die Erben des Dr. T sich durch Klageeinreichung der Gerichtsbarkeit des angerufenen Gerichts unterworfen hätten (vgl. Rn. 236 des Gutachtens), wäre die Zuständigkeit eines New Yorker Gerichts für eine gegen die Kläger gerichtete Klage der Erben gegeben.

Es ist auch nicht ersichtlich, dass ein New Yorker Gericht eine Entscheidung nach dem common law-Institut des forum non conveniens abgelehnt hätte. Für das Recht von New York ist insoweit Sec. 327(a) CPLR maßgeblich. Hiernach darf ein Gericht, wenn es der Auffassung ist, dass eine Klage im Interesse materieller Gerechtigkeit vor einem anderen Gericht gehört werden sollte, den Antrag einer Partei hin die Klage ganz oder teilweise unter angemessenen Bedingungen aussetzen oder abweisen; das Domizil oder der Wohnort im Forumstaat einer 
Partei des Rechtsstreits hindert das Gericht nicht daran, die Klage auszusetzen oder abzuweisen (Rn. 254 des Gutachtens). Die Darlegung und der Beweis der Gründe für eine Ablehnung der Entscheidung trotz bestehender Zuständigkeit obliegt der Partei, die sich auf forum non conveniens beruft (Rn. 255 des Gutachtens). Als Gründe anerkannt sind etwa Schwierigkeiten der Parteien, den Rechtsstreit vor dem angerufenen Gericht fortzuführen, das öffentliche Interesse einer Belastung des angerufenen Gerichts, mögliche Härten für den Beklagten oder die Nichtverfügbarkeit eines anderen Forums für die Klage (Rn. 255 des Gutachtens mwN). Bedeutsam kann aber auch sein, dass beide Parteien nicht im Gerichtsstaat ansässig sind oder sich die Geschehnisse, aus denen der Rechtsstreit erwachsen ist, in erster Linie in einer fremden Jurisdiktion zugetragen haben (Rn. 255 des Gutachtens unter Hinweis unter anderem auf Islamic Republic of Iran v. Pahlavi, 62 N.Y.2d 474, 467 N.E.2d 245 (248) (N.Y. 1984)). Das Gericht muss hierzu eine Abwägung der für und gegen den forum-non-conveniens sprechenden Tatsachen vornehmen (vgl. Rn. 257 des Gutachtens). Gemessen hieran geht der Senat davon aus, dass ein New Yorker Gericht einem non-conveniens-Einwand der Klägerin nicht folgt. In der Vergleichsentscheidung Vineberg v. Bissonnette (529 F.Supp.2d 300 (304) [D.R.I. 2007]) hatte das Gericht eine Abweisung der Klage auf Grundlage von forum non conveniens abgelehnt, obwohl in Deutschland bereits ein Verfahren in derselben Angelegenheit anhängig war und die Kläger keine US-Amerikaner waren. Es ist deshalb davon auszugehen, dass schon der Geschäftssitz der Beklagten in New York und eine Belegenheit des nämlichen Kunstwerkes am Geschäftssitz trotz der in Richtung Deutschland deutenden Interessen gegen einen forum-nonconveniens sprechen.

bb) Es erscheint demgegenüber unsicher, ob ein New Yorker Gericht auf Grundlage des Sachenrechtsstatuts oder des lex fori als Deliktsstatut entschieden hätte (vgl. Rn. $22 \mathrm{ff}, 90 \mathrm{ff}$ und 302 des Sachverständigengutachtens, Blatt 544 bis 740 der Akte). Das common law der anglo-amerikanischen Rechtsordnung und insoweit auch das Recht des US-Bundesstaates New York kennt zwar keinen dinglichen Herausgabeanspruch entsprechend dem deutschen Zivilrecht, jedoch sind Restitutionsansprüche anerkannt, die ihren Ursprung im Deliktsrecht finden (vgl. Rn. 5 des Sachverständigengutachtens mwN). Hierzu gehört auch die sog. Replevin-Klage, eine Klage auf Wiederbesitznahme von durch den Beklagten widerrechtlich an sich genommenem oder zurückgehaltenem Mobiliarvermögen, durch die der Kläger Sicherheit für das Gut gibt und das Gut besitzt, bis das Gericht entscheidet, wem es gehört (Rn. 5 des Sachverständigengutachtens). Im Recht von New York ist auch insoweit folgt der Senat dem Sachverständigen (aaO Rn. $7 \mathrm{ff}$ unter Hinweis u.a. auf folgende Fälle: Kunstsammlungen zu Weimar v. Elicofon, 536 F.Supp. 829 (845 f.) (E.D.N.Y. 1981); Warin v. Wildenstein \& Co., Inc., 2001 WL 1117493 [N.Y.Sup. 2001]) - ungeklärt, ob ein Replevin-Anspruch und seine Voraussetzungen dem Delikts- oder dem Sachenrechtsstatut zu entnehmen ist, wobei in beiden Fällen die Frage des anwendbaren Rechts nicht sicher zu beantworten ist.
(1) Die Gerichte von New York bestimmten das auf ein Delikt anwendbare Recht aufgrund einer Interessenabwägung, bei der die hinter den einander widersprechenden Regelungen stehenden rechtspolitischen Ziele bedeutsam sind; angewendet werden soll das Recht der Jurisdiktion, deren Interesse an der Regelung der konkreten Frage am größten ist (vgl. Rn. 23 des Gutachtens unter Hinweis auf Edwards v. Erie Coach Lines Co., 17 N.Y.3d 306, 952 N.E.2d 1033 (1036), 926 N.Y.S.2d 41 [N.Y. 2011]). Die Bestimmung des Rechts, dem verlustzuweisende Normen zu entnehmen sind, richtet sich nach den drei sogenannten Neumeier-Regeln (vgl. Rn. 25 des Gutachtens unter Hinweis auf Neumeier v. Kuehner, 31 N.Y.2d 121, 286 N.E.2d 454 (457 f.), 335 N.Y.S.2d 64 [N.Y. 1972]). Nach der ersten Regel sind verlustzuweisende Rechtssätze dem Recht der Jurisdiktion zu entnehmen, in der beide Parteien ihr domicile haben und - bei Verkehrsunfällen - das Fahrzeug gemeldet ist. Die zweite Regel führt zur Anwendung des Deliktsortsrechts, wenn dieses mit dem domicile einer der beiden Parteien zusammenfällt. Nach der dritten Regel ist hinsichtlich der übrigen Fälle das Deliktsortsrecht anzuwenden, außer, wenn gezeigt werden kann, dass die Verdrängung dieser normalerweise anwendbaren Regel die maßgeblichen Ziele des materiellen Rechts voranbringen wird, ohne das reibungslose Arbeiten des Mehrstaatensystems zu beeinträchtigen oder große Ungewissheit für die Prozessparteien auszulösen (vgl. Rn. $25 \mathrm{fdes}$ Gutachtens). Gemessen hieran würde ein New Yorker Gericht, soweit es den Replevin-Anspruch deliktsrechtlich qualifizieren würden, zwar voraussichtlich das Deliktsortsrecht anwenden. Damit ist aber gleichwohl nicht geklärt, wo nach Auffassung eines New Yorker Gerichts der loci delicti liegt. Nach vorgenanntem Maßstab ist - in Ermangelung eines nicht ersichtlichen gemeinsamen domicile der Klägerin einerseits und der Erben des Erblassers andererseits (vgl. hierzu Rn. 37 ff des Gutachtens) - entscheidend, ob ein New Yorker Gericht die unerlaubte Handlung in der Weigerung des Erwerbers eines gestohlenen Gutes sieht, dieses herauszugeben, oder ob auf den Ort der ungerechtfertigten Besitzerlangung abzustellen ist (Rn. $85 \mathrm{f}$ des Gutachtens). Im ersteren Fall wäre das Recht von New York als Tatortrecht maßgeblich, zumal die Klägerin dort ihren Geschäftssitz und damit ihr domicile im Sinne der zweiten Neumeier-Regel hat (Rn. 85 des Gutachtens). Im zweiten Fall wäre indes die Anwendung deutschen Rechts auf die Anspruchsvoraussetzungen eines Herausgabeanspruches maßgeblich (Rn. 86 des Gutachtens). Auch wenn eine gewisse Neigung der Gerichte besteht, zur Anwendung der lex fori zu gelangen, ist auch aus Sicht des Sachverständigen, der sich der Senat anschließt, nicht sicher, auf welche Handlung ein New Yorker Gericht abstellen würde. So ist etwa in der Entscheidung Federal Insurance Co. V. Fries (78 Misc.2d 805, 355 N.Y.S.2d 741 (747) (N.Y.City Civ.Ct 1974) von einer entsprechenden Interessenabwägung abgesehen worden (Rn. $87 \mathrm{f}$ des Gutachtens).

(2) Die Frage des anwendbaren Rechtes ist auch dann nicht sicher zu beantworten, wenn der Replevin-Anspruch sachenrechtlich zu qualifizieren ist. Das common law knüpfte die Übertragung von Rechten an beweglichen Sachen im Grundsatz an das domicile ihres Eigentümers (Rn. 90 des Gutachtens mwN). 
Hiernach wäre das deutsche Recht maßgeblich. Das nämliche Kunstwerk befand sich in den Zeitpunkten aller zu einer Eigentumsübertragung führenden Vorgänge bis einschließlich der Versteigerung vom 20. Mai 2000 in Deutschland (vgl. auch Rn. $99 \mathrm{ff}$ des Gutachtens). Nach neueren Entscheidungen ist indes die über Vertrags- und Deliktsstatut eingeführte Interessenabwägung (interest analysis) auch auf die Bestimmung des Sachenrechtsstatuts anwendbar (Rn. 91 ff des Gutachtens unter Hinweis u.a. auf: Bakalr v. Vavra, 619 F.3d 136 (143 f.) (C.A.2 (N.Y.) 2010); Schoeps v. Museum of Modern Art, 594 F. Supp.2d 461 (468) [S.D.N.Y. 2009]). Hiernach wären die Interessen der beteiligten Rechtsordnungen gegeneinander abzuwägen (Rn. 105 ff des Gutachtens). Zwar überwiegt regelmäßig das Interesse des Ortes, an dem sich die Sache physisch im Zeitpunkt der Eigentumsübertragung beziehungsweise des Entstehens eines Herausgabeanspruches befand (Rn. 106 des Gutachtens unter Hinweis auf das Restatement (Second) of Conflict of Laws (1971) $\S$ 244(2) sowie die Entscheidung Schoeps v. Museum of Modern Art, aaO). Da dies im Zeitpunkt der maßgeblichen Übertragungshandlung durch Zuschlag bei der Auktion am 20. Mai 2000 Deutschland war, wäre hiernach deutsches Recht maßgeblich. Indes steht dem der Grundsatz des New Yorker Rechts gegenüber, dass niemand von einem Dieb unbestreitbares Eigentum erwerben kann; Eigentümer sollen unabhängig von ihrem domicile geschützt werden, um die Ehrlichkeit und Fairness von Geschäften zu bewahren und zu verhindern, dass New York zu einem Marktplatz für gestohlene Güter wird (Rn. 110 des Gutachtens unter Hinweis u.a. auf: Kunstsammlung zu Weimar v. Elicofon, 536 F.Supp. 829 ((46) (D.C.N.Y. 1981); DeWeerth v. Baldinger, 658 F.Supp. 688 (692 f.) (S.D.N.Y. 1987); Bakalar v. Vavra, 619 F.3d 136 (144) (C.A.2 (N.Y.) 2010). Die Entscheidung Bakalar v. Vavra (aaO) betont ausdrücklich als entscheidend das Interesse New Yorks daran, nicht zum Marktplatz gestohlener Kulturgüter zu werden (Rn. 115 des Gutachtens). Gemessen hieran spricht aus Sicht des Senats vieles dafür, dass ein New Yorker Gericht, sollte es den Replevin-Anspruch als sachenrechtlich qualifizieren, zur Anwendung des lex fori gelangen würde.

cc) Vorgenannte Fragen dürfen indes trotz der Pflicht des Gerichts zur Ermittlung der Rechtslage nach ausländischem Recht (vgl. BGH, Urteil vom 30. April 1992 - IX ZR 233/90, BGHZ 118, 151, zitiert juris Rn. 25; vom 23. Juni 2003 - II ZR 305/01, NJW 2003, 2685, zitiert juris Rn. 9; Beschluss vom 21. Dezember 2011 - I ZR 144/09, TranspR 2012, 110, zitiert juris Rn. 11 ff) offen bleiben. Auch auf Grundlage des von der Klägerin herangezogenen New Yorker Rechts besteht kein Anspruch der Erben auf Herausgabe des Kunstwerkes, der gegen die Kläger hätte geltend gemacht werden können. Insbesondere besteht kein sogenannter Replevin-Anspruch, der voraussetzt, dass (1) die Erben rechtmäßige Eigentümer des Kunstwerkes sind, (2) das Kunstwerk dem Erblasser unrechtmäßig entwendet wurde und (3) die Klägerin unrechtmäßige Besitzerin des Kunstwerkes ist (Vineberg v. Bissonnette, 529 F.Supp. 2D 300 (306) [D.R.I.2007]).
(1) Der Erblasser war im Zeitpunkt der in Rede stehenden Auktion im Jahr 1937 Eigentümer des nämlichen Kunstwerkes. Dass seine Erben in eine etwaige fortbestehende Eigentümerstellung eingerückt wären, ist zwischen den Parteien unstreitig.

(2) Soweit der Replevin-Anspruch überdies eine unrechtmäBige Entwendung („unlawful taking“) des Gemäldes voraussetzt, die etwa angenommen wird, wenn dem ursprünglichen Eigentümer des Werkes dieses in einer einem Diebstahl oder einer offiziellen Beschlagnahme vergleichbaren Situation entwendet wird, liegt diese Voraussetzung nach Auffassung des Senats im konkreten Fall nicht vor. Zwar stehen die Methoden der Nationalsozialisten, den Verkauf von Kunst in Auktionen zu erzwingen, einem Diebstahl oder einen offiziellen Beschlagnahme gleich und erfüllen den Tatbestand der unrechtmäßigen Ansichnahme (Vineberg v. Bissonnette (529 F.Supp.2d 300 (307) [D.R.I. 2007]) und nachfolgend Vineberg v. Bissonnette, 548 F. 3d 50 [C.A.1 (R.I) 2008]). Ausnahmsweise verliert der Kunstgegenstand aber seinen Charakter als unrechtmäßig angenommenes Gut nach Auffassung des Senats, wenn eine Restitution dergestalt erfolgt ist, dass der rechtmäßige Eigentümer eines schon vor der Auktion zum Verkauf bestimmten Gegenstandes für den Gegenstand den Verkaufserlös und den aus dem Mindererlös resultierenden materiellen Schaden erhält.

Die von der Klägerseite angeführte Vergleichsentscheidung Vineberg v. Bissonnette (529 F.Supp.2d 300 (307) (D.R.I. 2007) steht dem nicht entgegen. Anders als im hiesigen Verfahren wurde dort in den Tatsachenfeststellungen festgehalten, dass der Erblasser aus dem Land floh, bevor er den Erlös aus dem erzwungenen Verkauf vereinnahmen konnte. Demgegenüber steht zur Überzeugung (§ 286 ZPO) des Senats auf Grundlage des hiesigen Parteivortrages und des Inhaltes der beigezogenen Akte im Entschädigungsverfahren 26 O 453/02 LG Düsseldorf fest, dass der Erblasser den Erlös aus der im Jahr 1937 erfolgten Auktion des nämlichen Kunstwerkes vereinnahmt hatte. Ausweislich der von den Beklagten in das Verfahren eingeführten Entscheidung des LG Düsseldorf vom 24. Februar 1964 hatte der Erblasser im vorgenannten Entschädigungsverfahren seinen Schaden unter Berücksichtigung eines erhaltenen Versteigerungserlöses errechnet. Nach den Angaben des Erblassers war nach Abzug der an das Auktionshaus zu entrichtenden Beträge vom Versteigerungserlös in Höhe von 4.800 Reichsmark (RM) für das nämliche Kunstwerk an ihn ein Betrag in Höhe von 4.230 RM ausgezahlt worden. Er hatte im Entschädigungsverfahren daher lediglich den Betrag geltend gemacht, um den der im Jahr 1937 bei der Auktion erzielte Auszahlungsbetrag hinter den damaligen Marktpreisen zurückgeblieben war, sowie weitere Nachteile, welche durch den Verkauf entstanden waren. Tatsachen, die die diese Angaben des Erblassers im Verfahren 260 454/62 des LG Düsseldorf in Frage stellen, hat die Klägerin demgegenüber nicht mehr vorgetragen.

(a) Ausweislich der Ausführungen des Sachverständigen Prof. Dr. N2 (Bl. 544 ff der Akte), die der Senat nachvollzieht und sich zu eigen macht, ist im Fallrecht sowohl von New York als 
auch anderen Bundesstaaten der USA bislang ungeklärt, unter welchen genauen Voraussetzungen ein privater Zwangsverkauf, der wie hier auf den Verfolgungsdruck des nationalsozialistischen Regimes erfolgt ist, einem Diebstahl gleichzusetzen ist (vgl. BI. 147 ff des Gutachtens). Es ist ungeklärt, ob ein unter dem Verfolgungsdruck des nationalistischen Regimes erfolgter Zwangsverkauf auch dann als Diebstahl zu werten ist, wenn für das Gemälde ein Kaufpreis an den ursprünglichen Eigentümer geflossen war (vgl. Rn. 274 des Sachverständigengutachtens unter Hinweis auf U.S. v. Portrait of Wally, 66 F. Supp.2d 232 (261 f) [S.D.N.Y. 2009]). Es ist in der US-amerikanischen Rechtsprechung anerkannt, dass ein Gegenstand seinen Charakter als Diebesgut (stolen property) verliert, wenn er seinem Eigentümer oder Stellvertreter zurückgegeben wird (vgl. hierzu Rn. 277 des Sachverständigengutachtens unter Hinweis auf U.S. v. Portrait of Wally, 105 F.Supp.2d 288 (293) (S.D.N.Y. 2000) mwN). Ob hierzu auch eine Restitution in Form von Schadensersatz ausreicht, ist indes nicht geklärt (Rn. 276 des Sachverständigengutachtens). Die vorgenannte Vergleichsentscheidung hatte sich zwar auch mit diesem Einwand auseinanderzusetzen, jedoch bestand in dem dort entschiedenen Fall kein Anhalt dafür, dass das Gemälde jemals Gegenstand eines Restitutionsverfahrens war (Rn. 276 des Sachverständigengutachtens). Ausweislich der Stellungnahme des Sachverständigen lassen die Ausführungen des Gerichts in dieser Vergleichsentscheidung es jedenfalls möglich erscheinen, dass die Gleichstellung eines verfolgungsbedingten Zwangsverkaufs im Falle einer Kaufpreiszahlung an den ursprünglichen Eigentümer entfällt (vgl. Rn. 274 ff des Gutachtens).

(b) Aus Sicht des Gerichts spricht zwar einiges dafür, dass allein der Erhalt des Erlöses einer erzwungenen Auktion allein die Gleichsetzung derselben mit einem Diebstahl oder einer offiziellen Beschlagnahme noch nicht entfallen lässt. Der Erlös entspricht in aller Regel im Falle einer Zwangsversteigerung nicht dem tatsächlichen Zeitwert des versteigerten Gegenstandes. Zudem folgt die Einordnung der erzwungenen Versteigerung als unrechtmäßige Ansichnahme aus einem durch Verfolgungsdruck des nationalsozialistischen Regimes bewirkten Zwang zum Verkauf. Die Auswirkungen dieses Zwangs bleiben selbst dann bestehen, wenn der rechtmäßige Eigentümer sogar einen angemessenen Preis für sein Kunstwerk erhält, aber - etwa als Sammler - nie beabsichtigt hatte, es zu veräußern. In diesen Fällen kann immer noch ein anzuerkennendes affektives oder kulturhistorisches Interesse an der Rückerlangung bestehen. Letzteres wäre insbesondere bei Kunstwerken aus Privathaushalten, Museen oder anderen Sammlungen denkbar.

(c) Im konkreten Fall handelte es sich jedoch um ein Gemälde, das zur Kunsthandlung des Erblassers gehörte und damit zum Verkauf bestimmt war. Dies stellt auch die Klägerin nicht in Frage. Sie geht selbst davon aus, dass das in Rede stehende Kunstwerk zum Bestand der Galerie T2 gehörte (Klageschrift vom 13. Juli 2012, Blatt 5 der Akte). Hiernach war es zu der Auktion im Jahr 1937 gekommen, weil dem Erblasser im Sommer 1937 aus Verfolgungsgründen untersagt worden war, seine
Kunsthandlung weiter zu betreiben, so dass ein Verkauf im ordnungsgemäßen Geschäftsverkehr nicht mehr erfolgen konnte. Der hierdurch entstandene materielle Verfolgungsschaden ist überdies auf Grundlage eines rechtsstaatlich ausgestalteten Entschädigungsverfahrens durch eine Entschädigungszahlung des deutschen Staates nach den Bestimmungen des Bundesentschädigungsgesetzes ausgeglichen worden. Das Bundesentschädigungsgesetz hat die Entschädigung der Opfer der nationalsozialistischen Verfolgung ( $§ 1$ BEG) zum Ziel, wobei bei der Besetzung der hierüber zu entscheidenden Entschädigungsgerichte gemäß § 208 Abs. 3 BEG dem Wesen der Wiedergutmachung in geeigneter Weise Rechnung zu tragen ist; der Vorsitzende oder einer der beiden Beisitzer soll dem Kreis der Verfolgten angehören. So hat die Entschädigungskammer des Landgerichts Düsseldorf mit Teilurteil vom 24. Februar 1964 (vgl. Urteil des LG Düsseldorf vom 24. Februar 1964 - 260 454/62, Blatt 276 ff der Akte) wegen des bei der Versteigerung hinsichtlich der betroffenen Kunstwerke insgesamt entstandenen verfolgungsbedingten Mindererlöses einen Schadensersatzbetrag in Höhe von 8.581,50 DM zuerkannt. Hinsichtlich des verfolgungsbedingt entstandenen Steuerschadens haben sich das Land Nordrhein-Westfalen und der Erblasser überdies im Vergleichswege auf die Zahlung eines weiteren Betrages von insgesamt 6.300 DM geeinigt. Dass der nach dem Bundesgesetz zur Entschädigung für Opfer der nationalsozialistischen Verfolgung (BEG) zuerkannte Verschleuderungsschaden nicht zur Auszahlung gelangt wäre, ist weder vorgetragen worden, noch besteht dazu Anhalt. Es ist vielmehr gerichtsbekannt, dass auf entsprechende Titel die zuerkannten Entschädigungen bezahlt wurden. Für eine Gleichstellung des Ergebnisses des durch Verfolgungsdruck des NS-Regimes im Jahr 1937 bewirkten Zwangsverkaufes beim Rechtsvorgänger der Beklagten mit einem Diebstahl besteht hiernach kein Raum mehr. Der durch den Zwangsverkauf eines im ordnungsgemäßen Geschäftsbetrieb ohnehin zum Verkauf bestimmten Kunstwerks verursachte Schaden und damit wenigstens das hierdurch erlittene materielle Unrecht sind ausgeglichen worden. Dass der Erblasser darüber hinaus an dem Werk ein besonderes affektives Interesse hatte, behauptet auch die Klägerin nicht.

(d) Hiergegen kann auch nicht eingewandt werden, dass die genannten Entschädigungsbeträge mit Blick auf die zwischenzeitlich eingetretene Inflation und auch die nunmehr fast 80jährige Entwicklung des Kunstmarktes weit hinter den heutigen Schätzwerten der betroffenen Kunstwerke zurückbleiben. Für die Entschädigung maßgeblich waren die Werte im Zeitpunkt der schädigenden Handlung und damit des Jahres 1937 unter Hinzurechnung eines bis zum Zeitpunkt der Entschädigungsentscheidung im Jahr 1964 erlittenen Nutzungsschadens (vgl. Seite 11 des Urteils des LG Düsseldorf vom 24. Februar 1964 - 26 O 454/62, Blatt 276 ff der Akte). Bei der Ermittlung der für die Entscheidung maßgeblichen Werte war die Entschädigungskammer, die den Erblasser als Verfolgten im Sinne von § 1 BEG anerkannt hatte, sogar ausdrücklich dem auf dem von ihm selbst eingeholten Privatgutachten des Sachverständigen Q (Blatt 287 ff der Akte, dort Blatt 294 der Akte) beruhenden Vor- 
trag des Erblassers vollständig gefolgt. Es hatte hierzu bei dem Kunstsachverständigen B ein gerichtliches Gutachten (Seite 21 f der Entschädigungsakte 260 454/62 LG Düsseldorf) eingeholt und sich auf dieser Grundlage den Wertangaben des Erblassers angeschlossen (vgl. Seite $8 \mathrm{f}$ des Urteils des LG Düsseldorf vom 24. Februar 1964 - 26 O 454/62, Blatt 276 ff der Akte). Hierbei war der Mindererlös durch einen Vergleich des vom Erblasser angegebenen Versteigerungserlöses mit einem möglichen Verkaufspreis, wie er in einem normalen Geschäftsbetrieb eines Kunsthauses im Jahre 1937 erzielt worden wäre, ermittelt worden. Auf Grundlage eines nach Maßgabe der Angaben des Privatgutachtens im ordnungsgemäßen Geschäftsverkehrs seinerzeit zu erzielenden Verkaufspreises von 6.000 RM machte der Erblasser deshalb als Verschleuderungsschaden für das Gemälde einen Differenzbetrag von 1.680 RM geltend, den die Entschädigungskammer auch anerkannte.

(3) Hiernach besteht angesichts des Fehlens einer unrechtmäßigen Entwendung des in Rede stehenden Kunstwerkes auch kein Raum für einen unrechtmäßigen Besitz der Klägerin. Sie hatte das Gemälde bei der Auktion im Jahr 2000 rechtmäßig erworben. Jedenfalls in diesem Zeitpunkt war die Erlangung des Werkes durch den Ersteigerer im Jahr 1937 angesichts der Weiterleitung des Verkaufserlöses aus der Versteigerung durch den Rechtsvorgänger der Beklagten und der in den 1960er-Jahren erfolgten Entschädigung nicht mehr unrechtmäßig.

dd) Unabhängig davon wäre ein Replevin-Anspruch nach dem amerikanischen Recht mangels ausreichender Such- und Restitutionsbemühungen des Erblassers und seiner Erben nach den Grundsätzen von laches (vgl. Rn. 223 ff des Gutachtens) verwirkt. Die billigkeitsrechtlichen Prinzipien der Verwirkung (laches) stehen der Geltendmachung eines ReplevinAnspruches entgegen, wenn der Anspruchsinhaber oder sein Rechtsvorgänger seinen Anspruch beziehungsweise die ihm zugrundeliegenden Tatsachen kennt oder kennen musste, aber dennoch ohne rechtfertigenden Grund sein Tätigwerden verzögert und dadurch die Gegenpartei benachteiligt (Rn. 302 des Gutachtens unter Hinweis auf Bakalar v. Vavra, 819 F.Supp.2d 293 (303 f.) (S.D.N.Y. 2011); Kamat v. Kurtha, 2008 WL 5505880 (S.D.N.Y. 2008)), wobei zur Kenntnis der anspruchsbegründenden Tatsachen auch das Wissen um die Identität des aktuellen Besitzers gehört (Rn. 225 des Gutachtens unter Hinweis u.a. auf In re Peters, 34 A.D.3d 29, 821 N.Y.S.2d 61 (N.Y.A.D. 1 Dept. 2006); Wertheimers v. Cirker's Hayes Storage Warehouse, 300 A.D.2d 117, 752 N.Y.S.2d 295 [N.Y.A.D. 1 Dept. 2002]). Erforderlich ist allerdings, dass der Eigentümer im Zeitraum zwischen dem Verschwinden und der Geltendmachung des Herausgabeanspruchs sorgfältig nach dem Kunstwerk gesucht und die Rückgabeforderung auch nach seiner Lokalisierung nicht unangemessen verzögert hatte (Rn. 227 des Gutachtens unter Hinweis u.a. auf Wertheimers v. Cirker's Hayes Storage Warehouse, aaO S. 297; De Weerth v. Baldinger, 836 F.2d 103 (110 ff.) (C.A.2 (N.Y.) 1987). Eine gewisse Verzögerung beim Tätigwerden hinsichtlich der Rückerlangung von unter dem nationalsozialistischen Regime oder während des Zweiten Weltkrieges verschwundener
Kulturgüter wird dabei mit Blick auf die ungewisse Eigentumslage und der politischen und wirtschaftlichen Unsicherheit nach dem Krieg als entschuldigt angesehen (Rn. 228 des Gutachtens unter Hinweis u.a. auf Bakalar v. Vavra, 819 F.Supp.2d 293 (305 f.) [S.D.N.Y. 2011]).

(1) Auf Grundlage des für den Senat maßgeblichen Sachund Streitstandes spricht im hiesigen Verfahren bereits viel für die Richtigkeit des Beklagtenvortrags, der Erblasser habe - im Gegensatz zu jenen Gemälden aus seiner Privatsammlung, die nach seiner Flucht im Jahr 1937 durch die Gestapo beschlagnahmt und verkauft worden waren - nach dem Krieg nicht nach den Gemälden aus der in Rede stehenden Versteigerung aus dem Jahr 1937 gesucht. Dem steht nicht entgegen, dass die Klägerin dem Vortrag mit dem Hinweis auf die Feststellungen der zitierten Vergleichsentscheidung (Vineberg v. Bissonnette (529 F.Supp.2d 300 [D.R.I. 2007]) entgegengetreten ist (vgl. insbesondere Schriftsatz vom 1. November 2012, Blatt 119 der Akte). Hiernach hatte der Erblasser nach Ende des zweiten Weltkrieges nach seinen Kunstwerken gesucht (Vineberg v. Bissonnette (529 F.Supp.2d 300 (303) [D.R.I. 2007]). Diese auf Grundlage des jeweiligen Parteivortrages im dortigen Verfahren getroffenen und damit für den Senat nicht bindenden Feststellungen unterscheiden indes schon nicht zwischen den Werken, die der Erblasser auf Verfolgungsdruck selbst dem Rechtsvorgänger der Beklagten zur Versteigerung übergeben hatte einerseits und den Werken, welche die Gestapo nach seiner Flucht aus Deutschland beschlagnahmt und veräußert hatte andererseits. Aus allgemein zugänglichen Quellen ist dem Senat demgegenüber als allgemeinkundige Tatsache (vgl. Hk-ZPO/Saenger, 5. Aufl., § 291 Rn. 3) bekannt, dass der Erblasser nach dem zweiten Weltkrieg durch Zeitungsanzeigen nach Kunstwerken gesucht hatte, die er nach der in Rede stehenden Versteigerung bei dem Rechtsvorgänger der Beklagten im Jahr 1937 als Restbestände seiner Sammlung eingelagert hatte und die sodann im Jahr 1939 von dem NS-Regime beschlagnahmt und versteigert worden waren (vgl. „Späte Gerechtigkeit für T“ unter http:// scriptore.de/spaete-gerechtigkeit-fuer-T2). Hinsichtlich der Kunstwerke aus der in Rede stehenden Versteigerung durch den Rechtsvorgänger der Beklagten ist solches allgemein zugänglichen Quellen - soweit für den Senat ersichtlich - nicht zu entnehmen. Überdies spricht auch das im Verfahren bekannt gewordene Verhalten des Erblassers hiergegen. Dieser hatte, wie gezeigt, im Jahr 1964 vor der Entschädigungskammer des Landgerichts Düsseldorf hinsichtlich der Kunstwerke aus der Versteigerung aus dem Jahr 1937 lediglich einen sogenannten Verschleuderungsschaden geltend gemacht (vgl. Urteil des LG Düsseldorf vom 24. Februar 1964 - 260 454/62, Blatt 276 ff, Blatt 294 [unter Nr. 185] der Akte), wobei ihm auch ein Anspruch auf Entschädigung des Verschleuderungsschadens zuerkannt wurde. Diese Entscheidung hatte der Erblasser nicht nur hingenommen, indem er hiergegen kein Rechtsmittel einlegte. Er hatte sich überdies hinsichtlich des mit seiner gegen das Land Nordrhein-Westfalen gerichteten Klage als weiteren Verfolgungsschaden geltend gemachten Steuerschadens mit dem Land gütlich geeinigt. Dass der Erblasser demgegenüber an den 
Rechtsvorgänger der Beklagten herangetreten wäre mit dem Anliegen auf Mithilfe bei der Rückerlangung der von diesem im Jahr 1937 versteigerten Kunstwerke etwa durch Bekanntgabe der persönlichen Daten der Ersteigerer oder dass der Erblasser den Rechtsvorgänger auf Schadensersatz in Anspruch genommen hätte, behauptet auch die Klägerin nicht. Das Bemühen des Erblassers richtete sich hiernach lediglich auf den Ersatz des verfolgungsbedingten Verschleuderungs- und Steuerschadens durch das Land Nordrhein-Westfalen und damit allein auf die Realisierung des wirtschaftlichen Wertes des in Rede stehenden Kunstwerkes und nicht auf die Rückerlangung des Werkes selbst. Allerdings ist zuzugestehen, dass dies Rückerlangungsbemühungen des Erblassers nicht schlechterdings ausschließt.

(2) Indes hat der Senat aus einem anderen Grund von der Richtigkeit des Vortrages der Beklagten zu fehlenden Suchbemühungen des Erblassers auszugehen. Die Anforderungen an den Vortrag der Parteien im hiesigen Verfahren unterliegen als Verfahrensrecht dem deutschen Recht (vgl. BGH, Beschluss vom 14. Oktober 1992 - XII ARZ 23/92, NJW-RR 1993, 130, zitiert juris Rn. 3; Palandt/Thorn, BGB, 75. Aufl., Einl. vor Art. 3 EGBGB Rn. 33; jeweils mwN). Die Beklagten sind hiernach zwar für die insoweit für sie günstige Tatsache einer Nichtverfolgung von Herausgabeansprüchen betreffend der Kunstwerke aus der Versteigerung durch den Rechtsvorgänger der Beklagten darlegungs- und beweisbelastet. Ein solcher Negativbeweis führt grundsätzlich auch nicht zu einer Änderung der Beweislast (vgl. BGH, Urteil vom 18. Februar 2009 - XII ZR 163/07, WM 2009, 2093, zitiert juris Rn. 19, 22; vom 22. Februar 2011 - XI ZR 261/09, WM 2011, 2130, zitiert juris Rn. 19; jeweils mwN). Jedoch trifft die Klägerin als Prozessgegnerin einer für eine negative Tatsache beweisbelasteten Partei eine sogenannte sekundäre Darlegungslast; dadurch soll eine unbillige Belastung der beweispflichtigen Partei vermieden werden (vgl. BGH, Urteil vom 24. März 2010 - XII ZR 175/08, BGHZ 185, 1, zitiert juris Rn. 20 mwN). Um die tatsächliche Schwierigkeit eines Nachweises negativer Tatsachen zu mildern, hat die damit belastete Partei daher in der Regel nur die Umstände zu widerlegen, die nach dem Vortrag der Gegenseite für die positive Tatsache, also für das Vorhandensein des streitigen Umstands, sprechen (BGH, Urteil vom 27. September 2002 - V ZR 98/01, WM 2003, 640, zitiert juris Rn. 7; vom 18. Februar 2009 - XII ZR 163/07, WM 2009, 2093, zitiert juris Rn. 20 f.; vom 22. Februar 2011, aaO Rn. 20). Der nicht beweisbelasteten Partei obliegt es deshalb, im Rahmen des ihr Zumutbaren die Behauptung der positiven Tatsachen aufzustellen, deren Unrichtigkeit sodann die beweisbelastete Partei nachzuweisen hat (BGH, Urteil vom 22. Februar 2011, aaO Rn. 20; Hk-ZPO/Saenger, $\S 286$ Rn. 92 ff; jeweils mwN). Dem wird der pauschale Hinweis der Klägerin auf die Ausführungen der zitierten Vergleichsentscheidung (Vineberg v. Bissonnette (529 F.Supp.2d 300 (303) [D.R.I. 2007]), nach welchen der Erblasser auf der Suche nach seinen Kunstwerken unter anderem im Jahr 1949 nach Europa gereist und in den Jahren 1948 und 1952 Anzeigen in Kunstzeitschriften geschaltet habe, nicht gerecht. Angesichts von der Beklagtenseite getroffenen Unterscheidung zwischen Werken, die von der Gestapo beschlagnahmt und veräußert worden waren, einerseits und denjenigen aus der in Rede stehenden Auktion andererseits wäre es erforderlich gewesen, konkrete Suchbemühungen zu benennen, die sich entweder auf alle Werke aus der vorgenannten Auktion bezogen oder auf das konkrete Bild. Die Klägerin gab auch nicht an, in welcher Ausgabe welcher Zeitschriften Annoncen welchen Inhalts aufgegeben wurden. Allein die pauschale Behauptung, der Erblasser habe nach dem Krieg nach seinen Kunstwerken gesucht, ist nicht ausreichend.

(3) Die hinsichtlich des hier in Rede stehenden Kunstwerkes unterbliebenen Rückerlangungsbemühungen des Erblassers beruhten auch nicht auf einer ungewissen Eigentumslage oder einer politischen und wirtschaftlichen Unsicherheit nach dem Krieg. Dies hat keine der Parteien behauptet. Nach Maßgabe des für den Senat aus vorgenannten Gründen maßgeblichen Vortrages begannen erst seine Erben mit der Suche nach dem Werk, wobei dieses schließlich im Jahr 2004, mithin knapp vier Jahre nach der hier in Rede stehenden Versteigerung des Jahres 2000, im Art Loss Register als gestohlen vermerkt wurde. Jedenfalls eine solche fast 60 Jahre umfassende Verzögerung ist nicht mehr entschuldigt. Durch die zeitlich gerechnet ab dem Jahr 1945 um fast 60 Jahre verzögerte Geltendmachung von Ansprüchen durch die Erben wird eine etwaige Gegenpartei benachteiligt. Die Erben setzen sich hierdurch in Widerspruch zu dem hier maßgeblichen Verhalten des Erblassers, der das Auktionsergebnis aus dem Jahr 1937 nach Erhalt einer Entschädigung als für ihn abgeschlossen behandelt hat. Sie verschlechtern durch dieses Verhalten die Rechtsposition einer Gegenpartei, indem sie deren Darlegungs- und Beweismöglichkeiten erschweren. Der Nachweis, dass für das in Rede stehende Kunstwerk erlittene Verfolgungsunrecht eine Entschädigung des Erblassers erfolgt ist und der Versteigerungserlös zur Auszahlung gelangte, kann nur deshalb geführt werden, weil die Akten der Verfahren nach dem Bundesentschädigungsgesetz aus zeithistorischen Gründen aufbewahrt werden.

c) Ein Rechtsmangel hinsichtlich des nämlichen Kunstwerkes folgt schließlich auch nicht aus seiner Beschlagnahme durch die US-Behörden nach Maßgabe der "Stipulation“ (Vereinbarung) vom 27./28. Mai 2009, in welcher der Geschäftsführer der Klägerin der Beschlagnahme durch die amerikanischen Behörden zustimmte und die Herausgabe des Gemäldes an die Erben vereinbarte (Anlage K12, 36 GA). Es ist in diesem Zusammenhang auch unbeachtlich, dass das Gemälde anschließend von den US-Behörden den Erben des Erblassers zurückgegeben wurde. Zwar können auch öffentlich-rechtliche Einziehungs- und Beschlagnahmebefugnisse einen Rechtsmangel begründen (vgl. BGH, Urteil vom 5. Dezember 1990 - VIII ZR 75/90, BGHZ 113, 106, zitiert juris Rn. 14; vom 18. Februar 2004 - VIII ZR 78/03, NJW 2004, 1802, zitiert juris Rn. 7). Voraussetzung ist jedoch, dass die Beschlagnahme tatsächlich ausgeübt wird, zu Recht erfolgt und den Verfall oder die Einziehung der Sache zur Folge haben kann (BGH, Urteil vom 18. Februar 2004, aaO Rn. 9 mwN). So soll der Verkäufer bei einer Beschlagnahme nach § 94 StPO (Sicherung von Beweismitteln) nicht haften, weil diese Maßnahme nur vorübergehender Natur ist und dieses Opfer dem Einzelnen im Interesse der Allgemeinheit an der Aufklärung von Straftaten 
auferlegt ist (vgl. Palandt/Weidenkaff, BGB, 75. Aufl., § 435 Rn. 13; OLG Köln, Beschluss vom 16. März 2010 - 22 U 176/09, n.v., zitiert juris Rn. 6; offen gelassen von BGH, Urteil vom 18. Februar 2004, aaO Rn. 11 ff). Diese Voraussetzungen liegen indes nicht vor. Es kann offen bleiben, ob hinsichtlich der Beschlagnahme eine Entsprechung des ausländischen Rechts in der deutschen Rechtsordnung erforderlich ist. Denn nach Vorgenanntem vermag eine Beschlagnahme einen Rechtsmangel nur zu begründen, wenn sie rechtmäßig erfolgt ist und zu einem dauerhaften Rechtsverlust führt, die Erben des Dr. T mithin nach US-amerikanischem Recht als die rechtmäßigen Eigentümer anzusehen sind. Dies ist aus vorstehenden Gründen allerdings gerade nicht der Fall. Die "Stipulation“ (Vereinbarung) vom 27./28. Mai 2009 geht, insoweit möglicherweise der Vergleichsentscheidung (Vineberg v. Bissonnette (529 F.Supp.2d 300 (307) [D.R.I. 2007]) folgend, von der durch den Inhalt der Entschädigungsakte 260 (Entsch.) 454/62 LG Düsseldorf widerlegten Annahme aus, der Erblasser habe die Erlöse aus der in Rede stehenden Versteigerung durch den Rechtsvorgänger der Beklagten im Jahr 1937 nicht erhalten.

2. Die Klägerin kann von den Beklagten auch keinen Schadensersatz unter dem Gesichtspunkt der bis zum Inkrafttreten des Schuldrechtsmodernisierungsgesetzes zum 1. Januar 2002 anerkannten Grundsätze der sogenannten culpa in contrahendo (Verschulden bei Vertragsverhandlungen, vgl. nur BGH, Urteil vom 20. Juni 1952 - V ZR 34/51, BGHZ 6, 330, zitiert juris Rn. 20 ff; vom 28. Januar 1976 - VIII ZR 246/74, BGHZ 66, 51, zitiert juris Rn. 8) verlangen.

a) Ein solcher Anspruch kann auch neben der Rechtsmängelhaftung nach § 434 BGB geltend gemacht werden (BGH, Urteil vom 6. April 2001 - V ZR 394/99, ZIP 2001, 1465, zitiert juris Rn. 9). Wirkt jemand bei Vertragsverhandlungen pflichtwidrig und schuldhaft durch unzutreffende Informationen auf die Willensbildung seines Vertragspartners ein und kommt es deshalb zum Abschluss eines wirksamen, aber für den Vertragspartner nachteiligen Vertrages, so verpflichtet dieses Verhalten nach den genannten Grundsätzen des Verschuldens bei Vertragsverhandlungen regelmäßig zum Schadensersatz (vgl. BGH, Urteil vom 14. März 1991 - VII ZR 342/89, BGHZ 114, 87, zitiert juris Rn. 12; vom 18. Juni 1997 - XII ZR 192/95, BGHZ 136, 102, zitiert juris Rn. 12). Die Voraussetzungen eines solchen Anspruches liegen indes nicht vor. Dem steht nicht entgegen, dass eine Pflichtverletzung nicht ausgeschlossen werden kann. Auch bei Vertragsverhandlungen, in denen die Parteien entgegengesetzte Interessen verfolgen, besteht für jeden Vertragspartner die Pflicht, den anderen Teil über Umstände aufzuklären, die den Vertragszweck des anderen vereiteln können und daher für seinen Entschluss von wesentlicher Bedeutung sind, sofern er die Mitteilung nach Treu und Glauben unter Berücksichtigung der Vertragsanschauung redlicherweise erwarten darf (BGH, Urteil vom 2. Februar 1996 - V ZR 239/94, BGHZ 132, 30, zitiert juris Rn. 17; vom 11. August 2010 - XII ZR 192/08, NJW 2010, 3362, zitiert juris Rn. 22; vom 1. Februar 2013 - V ZR 72/11, NJW 2013, 1807, zitiert juris Rn. 8; Palandt/Weidenkaff, BGB, 75. Aufl., § 433 Rn. 23; jeweils mwN). Zwar bestehen, wie gezeigt, gegen den gegenwärtigen Besitzer des Kunstwerkes gerichtete
Restitutionsansprüche auch nach US-amerikanischen Recht nicht. Eine Pflichtverletzungen kann gleichwohl darin erblickt werden, dass die Beklagte zu 1) trotz Kenntnis des Umstandes, dass der Erblasser seine Galeriebestände auf den verfolgungsbedingten Druck der Reichskulturkammer hatte versteigern lassen müssen, auf Nachfrage der Klägerin per Fax (Anlage K 4, Blatt 15 der Akte) unter anderem mitteilen ließ, dass die Herkunft des Gemäldes "sauber" sei. Gleiches gilt, soweit der Beklagte zu 2) telefonisch garantiert haben soll, dass es mit Blick auf die Auktion von 1937 keine Probleme mit dem Bild gebe, und dabei auch angegeben haben soll, dass die Galerie T2 keine jüdische Galerie gewesen sei. Die Klägerin hatte nämlich ein berechtigtes Interesse an der Kenntnis der näheren Umstände der ersten Versteigerung des Kunstwerkes im Jahr 1937. Sie war der Gefahr ausgesetzt, Restitutionsbemühungen der Erben abwehren und hierfür Aufwendungen tätigen zu müssen. Ebenso bestand ein anzuerkennendes Interesse der Klägerin, in der Öffentlichkeit nicht dem Vorwurf ausgesetzt zu werden, sich am Handel mit NS-Raubkunst zu beteiligen, und zwar unabhängig davon, ob der Vorwurf berechtigt oder unberechtigt ist. Solche Schäden macht die Klägerin jedoch nicht geltend. Sie erstrebt vielmehr den vollständigen Wertersatz des Gemäldes (sog. positives Interesse).

b) Überdies wäre ein Anspruch aus Verschulden bei Vertragsverhandlungen verjährt. Für das in Rede stehende Kaufgeschäft kommt die Höchstfrist von zehn Jahren gemäß § 199 Abs. 4 Satz 1 BGB zum Tragen. Diese kenntnisunabhängige Höchstfrist hat nicht erst im Jahr 2009 mit Herausgabe des Bildes zu laufen begonnen. Maßgeblicher Zeitpunkt war der Erwerb des Gemäldes im Jahr 2000, weil bereits zu dieser Zeit der Anspruch mit der etwaigen Pflichtverletzung und der Ersteigerung im Jahre 2000 entstanden war. Insoweit unterscheidet sich der Anspruch von demjenigen aus $\S 434, \S 440, \S 325$ Abs. 1 BGB a.F., bei welchem ein materieller Schaden erst zu dem Zeitpunkt entstanden war, zu welchem die Sache wegen des Rechtsmangels herausgegeben werden muss (vgl. OLG Hamm, Urteil vom 10.1.1975 - 20 U 197/74, NJW 1975, 2197). Auch wenn die Verjährungsfrist wegen der Übergangsvorschrift aus Art. $229 \S 6$ Abs. 4 EGBGB erst am 1. Januar 2002 begonnen hat, war sie gleichwohl vor Klageerhebung am 16. Juli 2012 mit Ablauf des Jahres 2011 abgelaufen und zwar unabhängig davon, ob der Beklagte zu 2) arglistig gehandelt hat oder nicht.

III.

Die Revision war nicht zuzulassen. Die Maßstäbe zur Ermittlung der in einem anderen Staat geltenden Rechte, der Gewohnheitsrechte und Statuten sind geklärt (vgl. BGH, Urteil vom 30. April 1992 - IX ZR 233/90, BGHZ 118, 151, zitiert juris Rn. 25; vom 23. Juni 2003 - II ZR 305/01, NJW 2003, 2685, zitiert juris Rn. 9; Beschluss vom 21. Dezember 2011 - I ZR 144/09, TranspR 2012, 110, zitiert juris Rn. 11 ff). Auf eine Verletzung von ausländischem Recht kann die Revision nicht gestützt werden $(B G H$, Beschluss vom 4. Juli 2013 - V ZB 197/12, BGHZ 198, 14, zitiert juris Rn. $15 \mathrm{ff} \mathrm{mwN}$ ).

(Das Urteil wurde redaktionell bearbeitet) 